\title{
Decreased Overall Survival of Transanal vs. Transabdominal Resection of Early Rectal Cancer in Treatment Naïve Patients: A National Cancer Data Base Study
}

\author{
Crystal Koerner, "*, Xi Sheng ${ }^{2}$, Yuan Liu², Theresa Gillespie ${ }^{3}$, Glen Balch ${ }^{4}$, Virginia Shaffer ${ }^{4}$, \\ Charles Staley $^{5}$, Jhanavi Srinivasan ${ }^{4}$, Patrick Sullivan ${ }^{4}$ \\ ${ }^{1}$ Department of Surgery, Emory University School of Medicine, Atlanta, USA \\ ${ }^{2}$ Department of Biostatistics and Bioinformatics, Emory University, Atlanta, USA \\ ${ }^{3}$ Department of Hematology \& Medical Oncology, Emory University School of Medicine and Winship Cancer Institute, Atlanta, USA \\ ${ }^{4}$ Division of Colon and Rectal Surgery, Emory University School of Medicine, Atlanta, USA \\ ${ }^{5}$ Division of Surgical Oncology, Emory University School of Medicine, Atlanta, USA
}

Email address:

Cpicke2@emory.edu (C. Koerner)

${ }^{*}$ Corresponding author

\section{To cite this article:}

Crystal Koerner, Xi Sheng, Yuan Liu, Theresa Gillespie, Glen Balch, Virginia Shaffer, Charles Staley, Jhanavi Srinivasan, Patrick Sullivan. Decreased Overall Survival of Transanal vs. Transabdominal Resection of Early Rectal Cancer in Treatment Naïve Patients: A National Cancer Data Base Study. Journal of Surgery. Vol. 7, No. 3, 2019, pp. 78-86. doi: 10.11648/j.js.20190703.16

Received: May 6, 2019; Accepted: June 6, 2019; Published: July 1, 2019

\begin{abstract}
Previous data from the National Cancer Database (NCBD) showed increasing rates of transanal local excision for early rectal cancer despite a lack of evidence supporting its oncologic adequacy. The aim of this study is to update national trends, compare overall survival, and determine factors associated with survival in patients with stage I rectal cancer. Survival of 15, 149 patients with stage I rectal adenocarcinoma were examined retrospectively from 2004-2012. The rate of local excision over this time period was sustained at $22 \%(20.88-24.9 \% ; \mathrm{p}=0.077)$. Five-year overall survival (OS) after transanal local excision was less than transabdominal standard resection $(76.6 \%$ vs. $80.7 \%$; $<0.0001)$. Lower 5 -year OS for transanal local excision was maintained with propensity score matching (HR 1.23; CI $(1.11-1.36$; $p<0.001$ ). Factors associated with decreased OS include positive margins, T2 tumors, tumors $>4 \mathrm{~cm}$, low volume centers, uninsured patients and increasing comorbidities. This is the first study of national data showing sustained use of transanal local excision over the past decade. Local excision has a lower 5-year OS compared to transabdominal standard resection. Transanal excision of early rectal cancer should be offered to select patients only after careful consideration of risk factors balanced against the decrease in overall survival.
\end{abstract}

Keywords: Transanal Local Excision, Stage 1 Rectal Cancer, Total Mesorectal Excision

\section{Introduction}

Each year, there are approximately 40,000 new cases of rectal cancer diagnosed in the United States [1]. Total mesorectal excision (TME) has been the standard of care for the surgical treatment of rectal cancer. Patient's undergoing TME for early stage rectal cancer have historically had excellent oncologic outcomes, with local recurrence rates from $0-7 \%$ and 5 -year disease free survival of $90 \%$ [2]. Transabdominal standard resection (SR), however, is associated with relatively high post-operative morbidity (30$68 \%$ ) and mortality (0-7\%) [2-4]. Given this relatively high morbidity and mortality, efforts have pursued whether less invasive procedures can maintain the same oncologic control while minimizing complications demonstrated by more localized procedures. Interest in local resection for early stage rectal cancer has been increasing due to ongoing 
concerns related to the high morbidity associated with TME. Initially used to treat patients who were poor surgical candidates or those with benign tumors, local excision (LE) is now being utilized with increasing frequency as definitive treatment for early stage rectal cancer. This has resulted in a two-fold increase in $\mathrm{T} 1$ and three-fold increase in $\mathrm{T} 2$ rectal tumors treated with local excision over the past decades [5].

Advantages of local excision include less post-operative morbidity, preservation of sphincter mechanism, and increased patient quality of life [5]. However, local excision for rectal cancer has been associated with increased local recurrence rates as high as $18 \%$ for $\mathrm{T} 1$ and $47 \%$ for $\mathrm{T} 2$ tumors [6-8]. Effects on survival have been less clear, with some studies concluding no effect on 5-year overall survival while others note a significant effect on overall and disease specific survival $[5,9]$.

Advances in technology and increasing interest in minimally invasive techniques have led to an increase in local excision of early rectal cancer, despite the lack of strong evidence supporting its oncologic adequacy. The American Society of Colorectal Surgeons (ASCRS) and the National Comprehensive Cancer Network (NCCN) support the use of local resection for $\mathrm{T} 1$ tumors less than $3 \mathrm{~cm}$ in size, accompanying less than $30 \%$ of the circumference of the bowel lumen, without high risk pathologic features (poorly differentiated tumors, lymphovascular invasion, positive margins, and greater than $1 \mathrm{~mm}$ submucosal depth) [10, 11]. The evidence supporting this recommendation is controversial. Patient selection likely plays an important role in the outcomes for patients receiving local excision, yet most studies do not standardize selection criteria. Understanding patient and tumor specific variables associated with survival are important for patient selection and influence patient outcomes. Additional studies are needed to clarify the subset of patients that may have acceptable oncologic outcomes with transanal local excision.

Our objective was to utilize contemporary National Cancer Data Base data to: 1) update recent national trends of local excision, 2) compare overall survival (OS) between the two techniques of interest, and 3) determine factors associated with OS in patients with stage I rectal cancer undergoing transanal local excision versus standard resection.

\section{Method}

\subsection{Data Source and Inclusion Criteria}

This study utilizes data from the National Cancer Data Base (NCDB), a joint program of the American Cancer Society and the Commission on Cancer of the American College of Surgeons (ACS). The NCDB is a nationwide database that captures $>75 \%$ of newly diagnosed cases of cancer in the US from over 1,500 ACS-accredited cancer programs in the US and Puerto Rico.

Patients greater than 18 years of age with stage 1 rectal adenocarcinoma diagnosed between 2004 and 2012 who received local excision or standard resection were initially included. Patients who received neoadjuvant therapy, palliative surgery, local tumor destruction, did not undergo surgery, and/or with prior cancer diagnosis were excluded from the study. After exclusions, the final analytical data set contained 15,149 patients. (Figure 1)

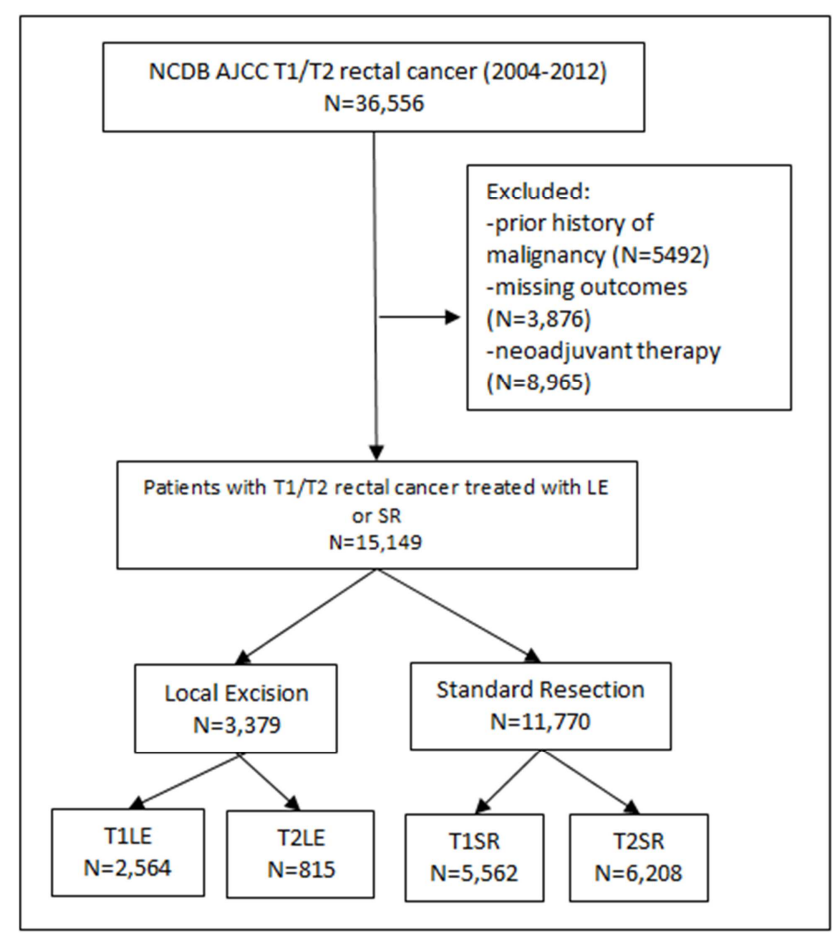

Figure 1. Study cohort after inclusion and exclusion criteria applied as selected from National Cancer Database. LE=Local excision, $S R=$ standard resection.

\subsection{Data Definitions}

Patient demographics were examined including age at diagnosis, race, primary payor, median income quartiles, education, comorbidities, and treating facility type, volume and geographic location. Defined comorbidities were included and used to calculate a Charleson-Deyo score. Facility type included academic/ research programs (provides postgraduate medical education), Comprehensive Community Cancer Programs (treating $>500$ cancer cases annually) and Community/ Integrated Network Cancer Programs (treating 100-500 cancer cases annually). Facility volume was defined as high volume ( $>25$ surgical cases per year) and low volume $(<25$ surgical cases per year) based upon quartiles of rectal surgery performed per year per facility during the study period.

Tumor characteristics were examined including tumor grade, tumor stage, tumor size and surgical margin status. Grade was reported as stated on the final pathology report. If the report listed more than one grade, the highest grade was coded, even if the highest grade was just a focus. T1 and T2 rectal tumors were included in the study based upon the AJCC pathologic $\mathrm{T}$ stage. Lymphovascular and perineural invasion were coded as present if identified in any portion of any specimen from the primary tumor. Surgical margin status was defined as positive or negative as reported on the final 
pathology report after resection of the primary tumor.

Local excision was defined as local tumor excision NOS and polypectomy NOS. Standard resection included partial proctectomy, coloanal anastomosis, total colectomy (may include portion of rectum), total proctocolectomy, proctectomy or coloproctectomy with resection of contiguous organs and proctectomy NOS. Patients who underwent local tumor destruction without pathologic specimen were excluded from the study. Overall survival was defined as months from date of surgery to death or last follow-up.

\subsection{Statistical Analysis}

Statistical analysis was conducted using SAS Version 9.3, and SAS macros or software developed at the Biostatistics and Bioinformatics at Winship Cancer Institute [12]. The significance level was set at 0.05 . Descriptive statistics for each variable were reported. The univariate association of each covariate with two cohorts of local excision and standard resection was assessed using the chi-square test for categorical covariates and ANOVA for numerical covariates. The prediction of local excision was further evaluated in multivariable logistic regression. The association with OS was assessed using Cox proportional hazards models and log-rank tests. A multivariable Cox proportional hazard model was built by a backward variable selection method applying an alpha $=.20$ removal criteria. The stratified analysis was conducted by including the interaction term between study cohorts and a stratified variable in a multivariable model and then hazard ratio was estimated for study cohorts in each level of the strata variable. KM plots were produced to compare the survival curves by subgroups along with log-rank p-value.
To further eliminate selection bias between two cohorts, a propensity score approach was used to balance the variables that predicted OS, named inverse probability of treatment weighting [13]. First a logistic regression model was fitted to estimate the probability that a patient could be assigned to a treatment based on his/her baseline covariates. This probability is called the propensity score. Then the study population was weighted by the inversed probability which was also stabilized to avoid extreme large weight and was normalized to represent the same sample size as the original sample. The balance diagnosis after weighting was carried by standardized difference with a value of $<0.15$, which was considered as negligible imbalance [14]. The treatment effects were estimated in the weighted sample by Cox proportional hazard model, and the weighted KM plots was generated accordingly $[15,16]$.

\section{Results}

\subsection{Trends in Surgical Approach}

Of the 15,149 patients included in our study, 11,770 (77.7\%) had SR while 3,379 (22.3\%) had LE. The trend of utilization of local excision for stage 1 rectal cancer was examined from 2004-2012. The annual rate of LE for stage I rectal cancer remained steady over the time period studied at $31 \%$ for $\mathrm{T} 1$ and $11 \%$ for $\mathrm{T} 2$ [(T1 29.1-33.8\%; $\mathrm{p}=0.35)(\mathrm{T} 2$ 9.97 - 14.5; $\mathrm{p}=0.11$ )]. (Figure 2) There was no predictable trend in the utilization of local excision versus standard resection over time with respect to facility type, geographic location, tumor grade or $\mathrm{T}$ stage.

\section{Trends of local excision 2004-2012}

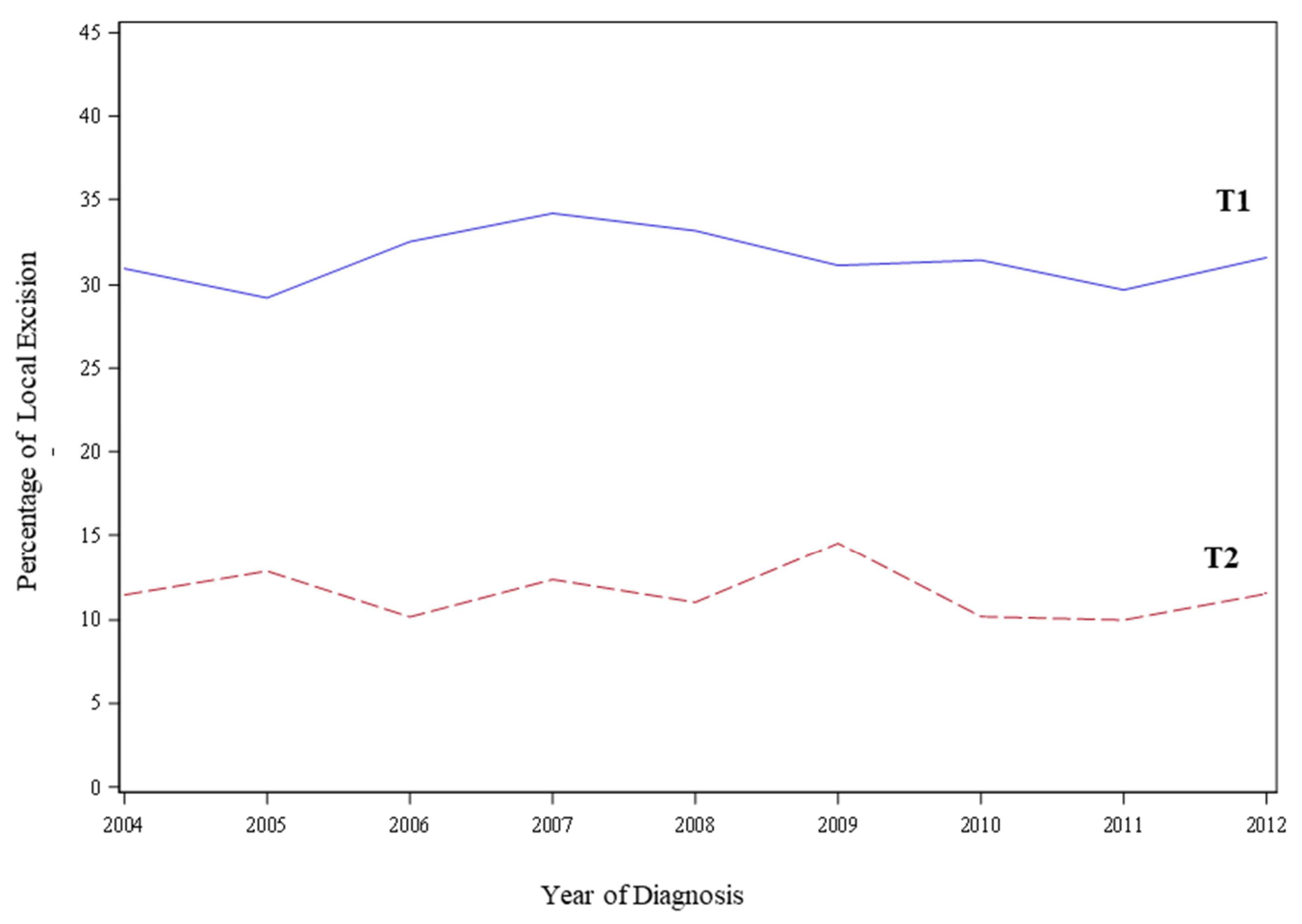

Figure 2. Percentage of patients with T1 (blue) and T2 (red) rectal cancer treated with local excision from 2004-2012. 
On univariate analysis, patients receiving local excision were more likely to be greater than 75 years of age, black/other race, uninsured, and had tumors less than $2 \mathrm{~cm}$. Patients with T1 tumors were much more likely to undergo local excision compared to T2 tumors $(31.55 \%$ vs $11.6 \% \mathrm{p}<0.001)$. Patients receiving care in high volume centers, academic, and community cancer programs were more likely to receive local excision compared to standard resection. On multivariate analysis, procedure selection was significantly associated with race, insurance status, tumor size, $\mathrm{T}$ stage, facility location and facility type. (Table 1)

Table 1. Multivariate Association of Treatment Groups (Local Excision vs Standard Resection) for Patients with Stage 1 Rectal Cancer.

\begin{tabular}{|c|c|c|c|c|}
\hline \multirow{2}{*}{ Covariate } & \multirow{2}{*}{ Level } & \multicolumn{3}{|c|}{ Surgery Type=Local Excision } \\
\hline & & Odds Ratio (95\% CI) & OR P-value & Type3 P-value \\
\hline \multirow{4}{*}{ Facility Type } & Unknown & $1.14(0.82-1.58)$ & 0.423 & \multirow{4}{*}{$<.001$} \\
\hline & Community/Integrated Network Cancer Program/Other & $0.95(0.84-1.06)$ & 0.363 & \\
\hline & Comprehensive Community Cancer Program & $0.74(0.67-0.81)$ & $<.001$ & \\
\hline & Academic/Research Program & - & - & \\
\hline \multirow{5}{*}{ Facility Location } & Unknown & - & - & \multirow{5}{*}{$<.001$} \\
\hline & West & $0.95(0.83-1.10)$ & 0.517 & \\
\hline & South & $1.17(1.04-1.31)$ & 0.009 & \\
\hline & Midwest & $0.96(0.85-1.09)$ & 0.565 & \\
\hline & Northeast & - & - & \\
\hline \multirow{3}{*}{ Race } & Other/Unknown & $1.12(0.93-1.35)$ & 0.239 & \multirow{3}{*}{0.002} \\
\hline & Black & $1.31(1.12-1.52)$ & $<.001$ & \\
\hline & White & - & - & \\
\hline \multirow{5}{*}{ Primary Payor } & Unknown & $0.57(0.38-0.85)$ & 0.006 & \multirow{5}{*}{$<.001$} \\
\hline & Medicare & $0.50(0.38-0.66)$ & $<.001$ & \\
\hline & Medicaid/Government Insurance & $0.57(0.41-0.80)$ & 0.001 & \\
\hline & Private Insurance & $0.53(0.40-0.69)$ & $<.001$ & \\
\hline & Not Insured & - & - & \\
\hline \multirow{3}{*}{ Charlson-Deyo Score } & 0 & $1.34(1.11-1.61)$ & 0.003 & \multirow{3}{*}{$<.001$} \\
\hline & 1 & $1.00(0.81-1.23)$ & 0.988 & \\
\hline & 2 & - & - & \\
\hline \multirow{2}{*}{ AJCC Pathologic T } & 2 & $0.39(0.36-0.43)$ & $<.001$ & \multirow{2}{*}{$<.001$} \\
\hline & 1 & - & - & \\
\hline \multirow{3}{*}{ Grade } & Cell Type Not Determined & $1.24(1.09-1.42)$ & 0.001 & \multirow{3}{*}{0.006} \\
\hline & Poorly Differentiated/Undifferentiated & $1.06(0.91-1.23)$ & 0.484 & \\
\hline & Well/Moderately Differentiated & - & - & \\
\hline \multirow{5}{*}{ Tumor Size (quartile) } & $>=0,<=2$ & $0.73(0.66-0.82)$ & $<.001$ & \multirow{5}{*}{$<.001$} \\
\hline & $>2,<=3$ & $0.44(0.39-0.50)$ & $<.001$ & \\
\hline & $>3,<=4$ & $0.39(0.33-0.45)$ & $<.001$ & \\
\hline & $>4,<=99$ & $0.29(0.25-0.34)$ & $<.001$ & \\
\hline & Unknown & - & - & \\
\hline
\end{tabular}

* Number of observations in the original data set $=15149$. Number of observations used $=15149$. ** Backward selection with an alpha level of removal of. 2 was used. The following variables were removed from the model: Median Income Quartiles 2000, Percent No High School Degree Quartiles 2000, Sex, Urban/Rural 2003, Diagnosis Year (quartile), and Number of surgical cases per year per facility.

\subsection{Overall Survival}

The 5-year OS after LE for stage I rectal cancer was significantly less than that for SR $(76.6 \%$ vs. $80.7 \%$; p $<0.0001)$ by log rank test. (Figure 3) 


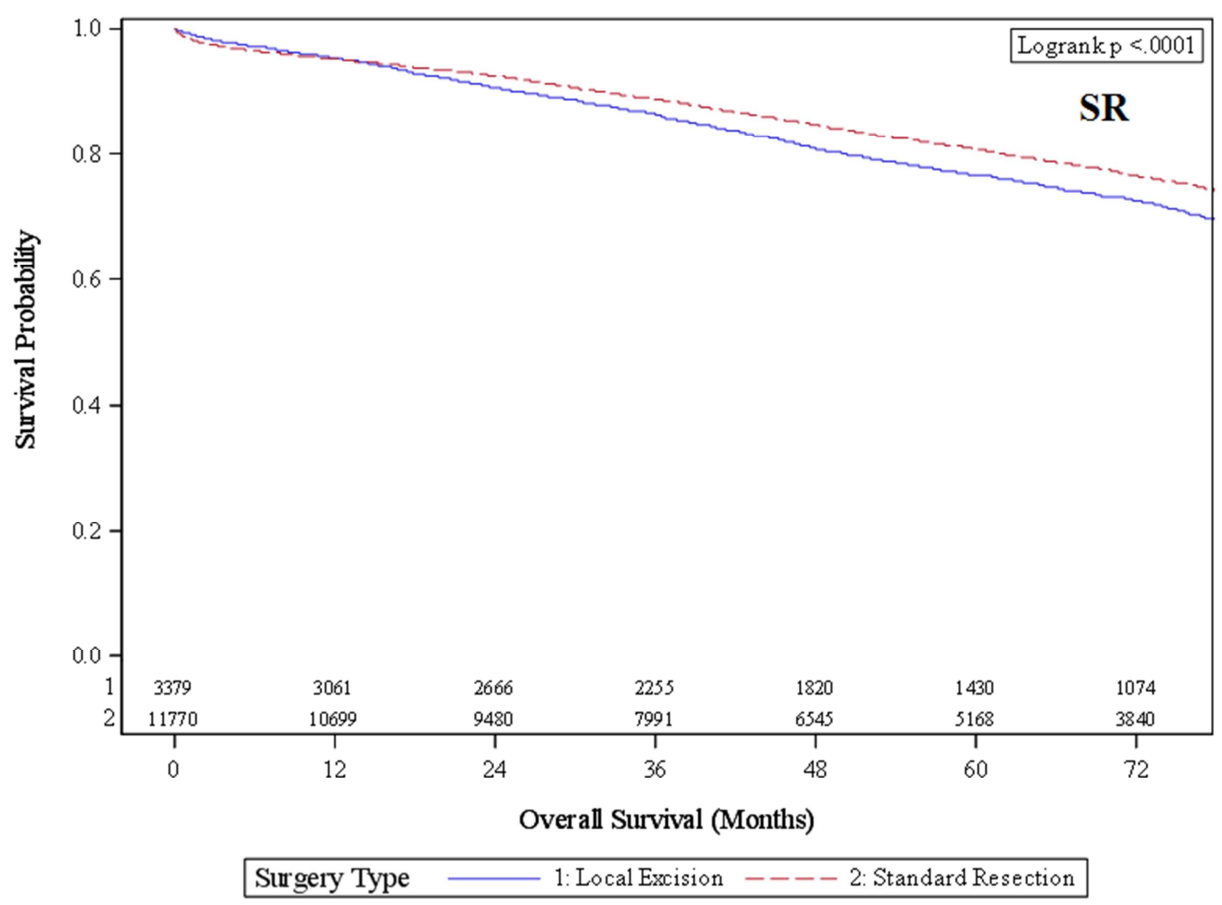

LE

Figure 3.Overall survival for stage 1 (T1 and T2) rectal cancer treated with local excision (LE) vs standard resection (SR).

When comparing patients with $\mathrm{T} 1$ tumors alone, patients undergoing LE had a significantly decreased OS in comparison to those undergoing SR $(80.5 \%$ vs. $86.0 \% ; p<0.001)$. This survival difference is more pronounced when comparing patients with T2 tumors undergoing LE vs SR (64.9\% vs. $76.1 \%$; $<<0.001)$. (Figure 4$)$

\section{T1}

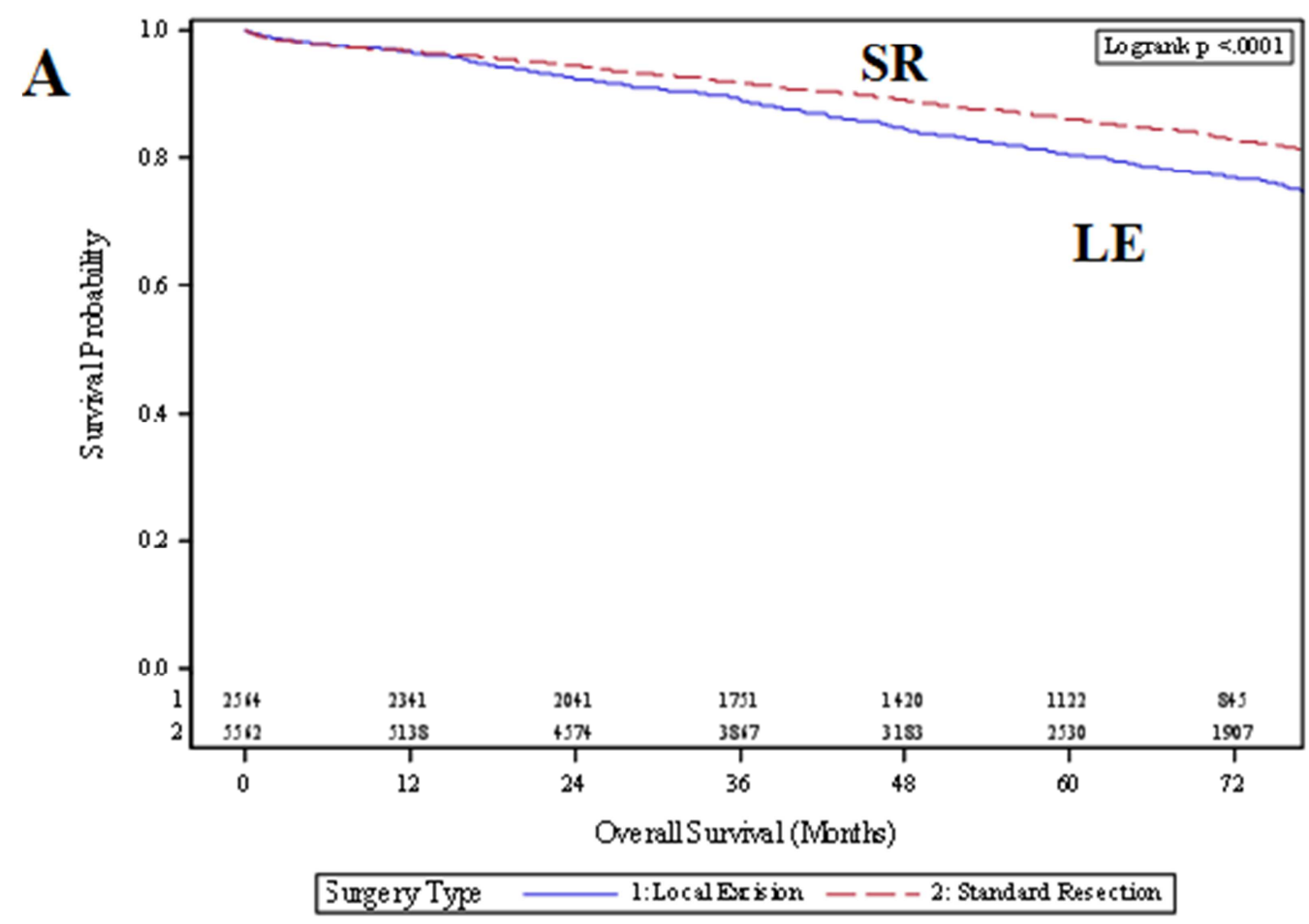



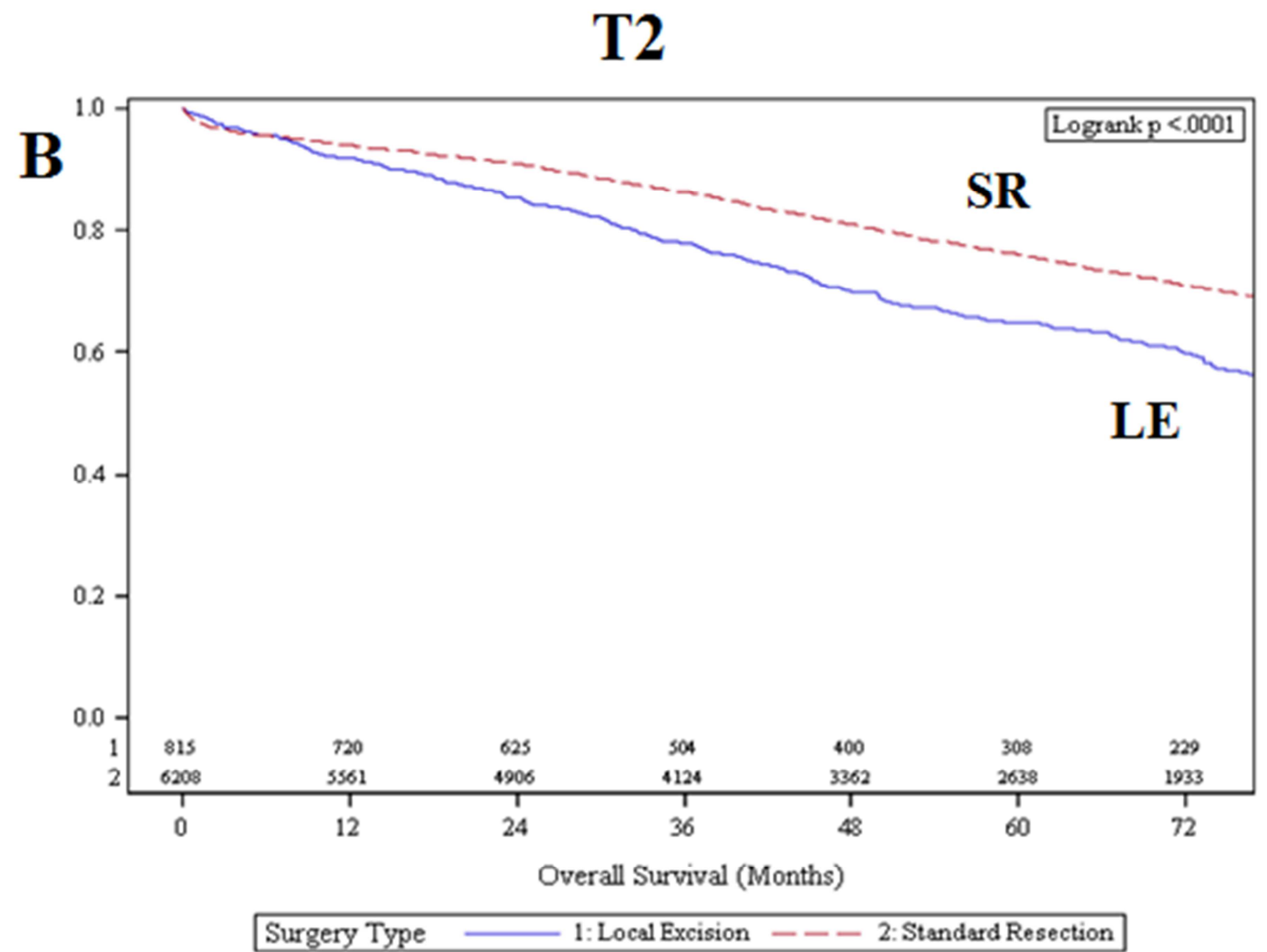

Figure 4. Overall survival for $T 1$ (A) and T2(B) rectal cancer treated with local excision (LE) versus standard resection (SR).

Lower 5 -year OS after LE compared to SR was maintained with propensity score matching (HR 1.23, CI (1.11-1.37); $\mathrm{p}<$ 0.001). When comparing T1 and T2 tumors alone, significantly lower 5-year OS was still noted for LE versus SR [T1; HR1.17 (CI 1.05-1.31); $\mathrm{p}=0.005$ : T2; HR 1.29 (CI 1.13-1.47); $\mathrm{P}<0.001]$. (Table 2)

Table 2. Overall Survival of Patients with $T 1$ (A) and T2 (B) Rectal Cancer Treated with Local Excision (LE) Versus Standard Resection (SR) with Propensity Score Matching.

$\mathrm{T} 1$

\begin{tabular}{lllll}
\hline \multirow{2}{*}{ Covariate } & \multirow{2}{*}{ Level } & N & \multicolumn{2}{l}{ Overall Survival (Months) } \\
\cline { 4 - 5 } & & Hazard Ratio (95\% CI) & HR P-value \\
\hline \multirow{2}{*}{ Surgery Type } & Local Excision & 2564 & $1.17(1.05-1.31)$ & 0.005 \\
& Standard Resection & 5562 & - & - \\
\hline
\end{tabular}

$\mathrm{T} 2$

\begin{tabular}{lllll}
\hline \multirow{2}{*}{ Covariate } & \multirow{2}{*}{ Level } & N & Overall Survival (Months) & HR P-value \\
\cline { 4 - 5 } & & & Hazard Ratio (95\% CI) & $<.001$ \\
\multirow{2}{*}{ Surgery Type } & Local Excision & 815 & $-29(1.13-1.47)$ & - \\
\hline
\end{tabular}

\subsection{Factors Associated with Overall Survival}

In the univariate analysis, age over 55, male sex, lower income, high comorbidity scores, uninsured status, receiving treatment in low volume centers and undergoing local excision were patient characteristics that were significantly associated with decreased OS. Tumor characteristics that were associated with a decreased OS include T2 tumors, tumors greater than $4 \mathrm{~cm}$ and positive surgical margins. All the above patient and tumor characteristics associated with survival were significant in the multivariate analysis, including undergoing local excision (HR 1.26; CI 1.16- 1.38; $\mathrm{p}<0.001)$. Age (HR 9.05; CI 7.98-10.27; $<<0.001)$ and comorbidities (HR 3.86; CI 3.48-4.29; $\mathrm{p}<0.001$ ) were the variables most predictive of poor survival. (Table 3 ) 
Table 3. Multivariate Association with Overall Survival Among Patients with Stage 1 Rectal Cancer Treated with Local Excision Versus Standard Resection.

\begin{tabular}{|c|c|c|c|c|}
\hline \multirow{2}{*}{ Covariate } & \multirow{2}{*}{ Level } & \multicolumn{3}{|l|}{ Overall Survival (Months) } \\
\hline & & Hazard Ratio (95\% CI) & HR P-value & Type3 P-value \\
\hline \multirow{3}{*}{ Surgery Type } & Local Excision & $1.26(1.16-1.38)$ & $<.001$ & \multirow{3}{*}{$<.001$} \\
\hline & Standard Resection & - & - & \\
\hline & Unknown & $2.20(1.30-3.72)$ & 0.003 & \\
\hline \multirow{3}{*}{ Facility Type } & Community/Integrated Network Cancer Program/Other & $1.05(0.95-1.16)$ & 0.354 & \multirow{3}{*}{0.021} \\
\hline & Comprehensive Community Cancer Program & $1.06(0.97-1.15)$ & 0.188 & \\
\hline & Academic/Research Program & - & - & \\
\hline \multirow{3}{*}{$\begin{array}{l}\text { Number of surgical cases } \\
\text { per year per facility }\end{array}$} & Low Volume & $1.12(1.04-1.20)$ & 0.003 & \multirow{2}{*}{0.003} \\
\hline & High Volume & - & - & \\
\hline & Other/Unknown & $0.71(0.58-0.88)$ & 0.001 & \multirow{3}{*}{$<.001$} \\
\hline \multirow[t]{2}{*}{ Race } & Black & $1.13(0.99-1.30)$ & 0.074 & \\
\hline & White & - & - & \\
\hline \multirow{2}{*}{ Sex } & Male & $1.36(1.27-1.46)$ & $<.001$ & \multirow{2}{*}{$<.001$} \\
\hline & Female & - & - & \\
\hline \multirow{5}{*}{ Primary Payor } & Unknown & $0.50(0.35-0.72)$ & $<.001$ & \multirow{5}{*}{$<.001$} \\
\hline & Medicare & $0.55(0.43-0.72)$ & $<.001$ & \\
\hline & Medicaid/Government Insurance & $0.90(0.66-1.22)$ & 0.479 & \\
\hline & Private Insurance & $0.45(0.35-0.58)$ & $<.001$ & \\
\hline & Not Insured & - $\quad 1 \quad-2$ & - & \\
\hline \multirow{5}{*}{$\begin{array}{l}\text { Median Income } \\
\text { Quartiles } 2000\end{array}$} & $\$ 46,000+$ & $0.63(0.53-0.74)$ & $<.001$ & \multirow{5}{*}{$<.001$} \\
\hline & $\$ 36,000-\$ 45,999$ & $0.66(0.56-0.79)$ & $<.001$ & \\
\hline & $\$ 30,000-\$ 35,999$ & $0.66(0.55-0.79)$ & $<.001$ & \\
\hline & $<\$ 30,000$ & $0.79(0.65-0.95)$ & 0.011 & \\
\hline & Unknown & - & - & \\
\hline \multirow{3}{*}{ Charlson-Deyo Score } & 2 & $2.62(2.36-2.91)$ & $<.001$ & \multirow{3}{*}{$<.001$} \\
\hline & 1 & $1.49(1.37-1.61)$ & $<.001$ & \\
\hline & 0 & - $\quad 1 \quad-2$ & - & \\
\hline \multirow{3}{*}{ AJCC Pathologic T } & 2 & $1.32(1.22-1.43)$ & $<.001$ & \multirow{2}{*}{$<.001$} \\
\hline & 1 & - $\quad 1 \quad-2$ & & \\
\hline & Well/Moderately Differentiated & $1.18(1.01-1.38)$ & 0.032 & \multirow{3}{*}{0.015} \\
\hline \multirow[t]{2}{*}{ Grade } & Poorly Differentiated/Undifferentiated & $1.33(1.09-1.61)$ & 0.004 & \\
\hline & Cell Type Not Determined & - & - & \\
\hline \multirow{2}{*}{ Surgical Margin } & Positive/Unknown & $1.41(1.23-1.62)$ & $<.001$ & \multirow{2}{*}{$<.001$} \\
\hline & Negative & - & - & \\
\hline \multirow{5}{*}{ Tumor Size (quartile) } & Unknown & $1.01(0.90-1.13)$ & 0.893 & \multirow{5}{*}{0.041} \\
\hline & $>4,<=99$ & $1.13(1.01-1.26)$ & 0.036 & \\
\hline & $>3,<=4$ & $1.01(0.90-1.13)$ & 0.903 & \\
\hline & $>2,<=3$ & $0.97(0.86-1.08)$ & 0.552 & \\
\hline & $>=0,<=2$ & - & - & \\
\hline
\end{tabular}

$*$ Number of observations in the original data set $=15149$. Number of observations used $=15149$. $* *$ Backward selection with an alpha level of removal of.20 was used. The following variables were removed from the model: Facility Location, Percent No High School Degree Quartiles 2000, Urban/Rural 2003, and Diagnosis Year (quartile).

Overall survival with respect to margin status and surgical type was examined. Patients with positive margins and T1 tumors resected locally had a lower 5-year OS compared to patients with negative margins ( $71 \%$ vs $81.9 \%$; p <0.001). This trend was not seen with patients with $\mathrm{T} 2$ tumors (SR+ margins $85.1 \%$ vs SR- margins $86.1 \%$; $p<0.001$ ). Of the 3,379 patients undergoing LE, $502(15 \%)$ had positive margins, while $259(2.2 \%)$ of the 11,770 patients undergoing SR had positive margins. Factors associated with positive margins include undergoing LE, low volume centers, age $>55$, black race, lower socioeconomic status, T1 tumors and tumors $<2 \mathrm{~cm}$.

\section{Discussion}

This study is the first to show consistent trends over time in the use of LE for treatment of stage 1 rectal cancer nationally. This trend may be a result of the growing literature that demonstrates the survival benefit of standard resection over local excision. This is the first study to our knowledge to demonstrate a higher 5-year OS for patients undergoing SR compared to patients treated with LE with a propensity score matched sample. Patient and tumor specific variables play an important role in operative planning and patient counseling. Factors associated with a lower overall survival included: age $>55$, male sex, multiple comorbidities, uninsured status, T2 tumors, larger tumors and $\mathrm{R} 1$ resections.

This study utilizes updated National Cancer Data Base data to demonstrate consistent use of LE in the treatment of stage 1 rectal cancer. Previous NCDB studies demonstrated an increase in the treatment of early rectal cancer with transanal LE, nearly two-fold for T1 and three-fold for T2 tumors from 1998-2003 [5]. A follow-up NCDB study analyzing trends in LE also demonstrated an increase in the rates of LE for stage 1 rectal cancer from 
1998-2010 [18]. The plateau in LE as definitive treatment for stage 1 rectal cancer may be a result of the growing literature that cites lower OS and increased local recurrence rates compared with transabdominal SR. In addition, our study excluded patients that received neoadjuvant therapy, which totaled 8,965 patients. It has been noted in prior studies that approximately $30-50 \%$ of patients with $\mathrm{T} 2$ tumors receiving $\mathrm{LE}$ received neoadjuvant therapy [17]. This may explain the discordance with our data and prior studies of the NCDB citing an increase in the use of LE from 1998-2010 [18]. It was important to exclude this subset of patients as our focus was on those patients who received surgery alone.

Overall survival for patients undergoing LE for stage 1 rectal cancer is lower than those treated with SR $(76.6 \%$ vs. $80.7 \% ; \mathrm{p}<0.0001)$. Our results are concordant with prior large retrospective studies. A Surveillance, Epidemiology, and End Results (SEER) study from 2013 concluded decreased $\mathrm{OS}$ in patients receiving $\mathrm{LE}$ of $\mathrm{T} 1$ and $\mathrm{T} 2$ rectal tumors versus SR (HR 1.26; T1, HR 1.71; T2) [9]. In addition, an NCDB study from 2013 also demonstrated a lower OS for T1 and T2 tumors undergoing LE alone [17]. Our study demonstrated a decrease in overall survival with LE vs SR with a propensity score matched sample, when controlling for patient and tumor specific variables that were independent predictors of survival. This study timeframe includes a period of innovation and increasing familiarity with transanal techniques, including transanal endoscopic microsurgery (TEM), transanal minimally invasive surgery (TAMIS) [18] and traditional transanal excision. These techniques have been shown in the literature to have variable outcomes when compared to one another. In a recent metanalysis, overall survival, disease free survival and distant metastasis rates did not differ between TEM and TME [19]. However, large series comparing TAMIS, TEM, and traditional TAE to TME for patients with early stage rectal cancer are lacking. The NCDB does not yet discriminate between these surgical techniques, resulting in heterogenous transanal operative techniques that are surgeon dependent and may affect applicability. In addition, our study is limited in that the NCDB does not provide data related to recurrence, additional chemotherapy or reoperation which would affect overall survival.

Patient and tumor specific factors have been shown to affect survival in patients with stage 1 rectal cancer undergoing local excision. This study demonstrates that age and comorbidities were most strongly associated with a lower overall survival. Tumor specific variables that were significant included $\mathrm{T}$ stage, larger tumors and positive surgical margins. Factors associated with positive margins included undergoing local excision, low volume centers, older age, black race, lower socioeconomic status, T1 tumors and tumors $<2 \mathrm{~cm}$. The above factors were more strongly associated with receiving local excision and may be attributable to the higher $\mathrm{R} 1$ ( $15 \%$ vs. $2.2 \%)$ resection rate with LE. R1 resection of T2 tumors did not translate to lower overall survival compared with R0 resections. This may reflect adjuvant therapy or salvage operations performed after initial LE. In a 2016 meta-analysis, TME after LE for T1/T2 rectal cancers had a 5-year overall survival from 86$94 \%$ [20].

Importantly, this study excluded those patients who received neoadjuvant therapy, as neoadjuvant therapy is being increasingly used for T2 tumors prior to local excision. Recent prospective and retrospective data have shown comparable disease-free survival, overall survival and local recurrence rates between transanal LE and TME in patients receiving neoadjuvant chemoradiation [21, 22]. In addition, the ACOSOG Z6041 published preliminary results demonstrating a high percentage of complete pathologic response and negative margin rate for those patients receiving neoadjuvant therapy followed by LE [23]. Our focus in this study was on treatment naïve patients who did not undergo neoadjuvant therapy or prior surgical intervention. Additional large series are needed to determine long term outcomes of patients with $\mathrm{T} 2$ tumors undergoing neoadjuvant therapy followed by transanal local excision.

The key findings of this study, in concordance with prior literature, suggest a significant survival benefit for patients with early stage rectal cancer treated with SR compared to transanal LE. Patient preferences and decreased morbidity of LE should be balanced carefully with the decrease in overall survival. Patients with T2 tumors should be counseled regarding the significant decrease in overall survival with treatment with LE alone. Patient and tumor specific factors should be carefully considered when offering a patient with early rectal cancer treatment with LE.

Important limitations of this study include the NCDB's lack of long-term follow-up data for patients that received additional chemotherapy or reoperation for an $\mathrm{R} 1$ resection or recurrence. Favorable tumor characteristics including well differentiated tumors, lack of perineural or lymphovascular invasion have been important in the criteria for patient selection for local excision in accordance with the NCCN and ASCRS guidelines. The NCDB did not collect this information routinely until 2010 , analyses were unable to be performed due to the lack of sufficient information. In addition, the NCDB does not capture cancer specific variables to draw conclusions regarding disease free or cancer specific survival. Further studies examining the oncologic adequacy of LE for low risk T1 tumors with favorable histology are needed to further characterize optimal patient selection for LE.

\section{Conclusion}

Over the past decade, the proportion of patients receiving local excision for stage 1 rectal cancer has remained steady at around $22 \%$. This may reflect the growing literature that has emerged recently supporting the oncologic superiority of standard resection, given that prior studies demonstrated an increase in the rates of LE from 1998-2010. Even with advances and increased familiarity with transanal excision techniques, patients 
with early stage rectal cancer treated with LE have a lower 5-year overall survival. Importantly, this study demonstrated a decrease in overall survival with LE vs SR with a propensity score matched sample, when controlling for patient and tumor specific variables that were independent predictors of survival. Thus, transanal local excision of stage I rectal cancer should be offered to select patients only after careful consideration of risk factors balanced against the decrease in overall survival.

\section{References}

[1] Howlader N, Noone AM, Krapcho M, et al. SEER Cancer Statistics Review, 1975-2014. National Cancer Institute. Bethesda, MD, https://seer.cancer.gov/csr/1975_2014/

[2] Althumairi AA, Gearhart SL. Local excision for early rectal cancer: transanal endoscopic microsurgery and beyond. J Gastrointest Oncol 2015; 6: 296-306.

[3] Elmessiry MM, Van Koughnett JAM, Maya A, et al. Local excision of $\mathrm{T} 1$ and $\mathrm{T} 2$ rectal cancer: proceed with caution. Colorectal Dis 2014; 16: 703-709.

[4] Garcia-Aguilar J, Mellgren A, Sirivongs P, et al. Local excision of rectal cancer without adjuvant therapy: a word of caution. Ann Surg 2000; 231: 345-351.

[5] You YN, Baxter NN, Stewart A, Nelson H. Is the increasing rate of local excision for stage I rectal cancer in the United States justified?: a nationwide cohort study from the National Cancer Database. Ann Surg 2007; 245: 726-733.

[6] Bentrem DJ, Okabe S, Wong WD, et al. T1 Adenocarcinoma of the Rectum: Transanal Excision or Radical Surgery? Annals of Surgery 2005; 242: 472-479.

[7] Rothenberger DA1, Garcia-Aguilar J. Role of local excision in the treatment of rectal cancer. Semin Surg Oncol 2000; 19: 367-75.

[8] Mellgren A1, Sirivongs P, Rothenberger DA, et al. Is local excision adequate therapy for early rectal cancer? Dis Colon Rectum 2000; 43: 1064-71.

[9] Bhangu A, Brown G, Nicholls RJ, et al. Survival outcome of local excision versus radical resection of colon or rectal carcinoma: A Surveillance, Epidemiology, and End Results (SEER) population-based study. Ann Surg 2013; 258: 563569.

[10] National Comprehensive Cancer Network. NCCN guideline updates: colon and rectal cancers, version 1.2004. J Natl Compr Canc Netw 2004; 2: 284-5.

[11] Monson JRT, Weiser MR, Buie WD, et al. Standards Practice
Task Force of the American Society of Colon and Rectal Surgeons. Practice parameters for the management of rectal cancer. Dis Colon Rectum 2013; 56:535-550.

[12] Nickleach D, Liu Y, Shrewsberry A, Ogan K, Kim S, Wang Z. SAS ${ }^{\circledR}$ Macros to Conduct Common Biostatistical Analyses and Generate Reports. SESUG 2013: The Proceeding of the SouthEast SAS User Group

[13] Austin PC, Grootendorst P, Anderson GM. A comparison of the ability of different propensity score models to balance measured variables between treated and untreated subjects: A Monte Carlo study Stat Med 2007; 26: 734-753.

[14] Austin PC, Stuart EA. Moving towards best practice when using inverse probablility of treatment weighting (IPTW) using propensity score to estimate casual treatment effects in observational studies. Stat Med 2015. 34: 3661-79.

[15] Cole SR, Hernan MA. Adjusted survival curves with inverse probability weights. Comput Methods Programs Biomed 2004; 75: 45-9.

[16] Parsons LS. Reducing bias in a propensity score matched-pair sample using greedy matching techniques. SAS SUGI 2001; 26: $214-226$.

[17] Stitzenberg KB, Sanoff HK, Penn DC, et al. Practice Patterns and Long-Term Survival for Early-Stage Rectal Cancer. Journal of Clinial Oncology 2013; 31: 4276-4282.

[18] Gill S, Stetler JL, Patel A, et al. Transanal Minimally Invasive Surgery (TAMIS): Standardizing a Reproducible Procedure. J Gastrointest Surg 2015; 19: 1528-36.

[19] Guerrieri M, Gesuita R, Ghiselli R, et al. Treatment of rectal cancer by transanal endoscopic microsurgery: Experience with 425 patients. World Journal of Gastroenterology 2014; 20: 9556-63.

[20] Borstlap WA, Coeymans TJ, Tanis PJ, et al. Meta-analysis of oncological outcomes after local excision of pT1-2 rectal cancer requiring adjuvant (chemo)radiotherapy or completion surgery. Br J Surg 2016; 103: 1105-16.

[21] Lezoche G, Baldarelli M, Guerrieri M, et al. A prospective randomized study with a 5-year minimum follow-up evaluation of transanal endoscopic microsurgery versus laparoscopic total mesorectal excision after neoadjuvant therapy. Surg Endosc 2008 Feb; 22: 352-8.

[22] Nair RM, Siegel EM, Chen DT, et al. Long-tern results if tranasanal excision after neoadjuvant chemoradiation for T2 and T3 adenocarcinomas of the rectum. J Gastrointest Surg 2008 Oct; 12: 1797-805.

[23] Garcia-Aguilar J, Shi Q, Thomas C, et al. A Phase II Trial of Neoadjuvant Chemoradiation and Local Excision for T2N0 Rectal Cancer: Preliminary Results of the ACOSOG Z6o41 Trial. Ann Surg Onc 2012 Feb; 19: 384-391. 